\title{
One-Step Fabrication of Hierarchically Structured Silicon Surfaces and Modification of Their Morphologies Using Sacrificial Layers
}

\author{
Seong J. Cho, ${ }^{1}$ Se Yeong Seok, ${ }^{1}$ Jin Young Kim, ${ }^{1}$ Geunbae Lim, ${ }^{1,2}$ and Hoon Lim ${ }^{3}$ \\ ${ }^{1}$ Department of Mechanical Engineering, Pohang University of Science and Technology (POSTECH), \\ Pohang 790-784, Republic of Korea \\ ${ }^{2}$ Department of Integrative Bioscience and Biotechnology, Pohang University of Science and Technology (POSTECH), \\ Pohang 790-784, Republic of Korea \\ ${ }^{3}$ Department of Emergency Medicine, Soon Chun Hyang University Hospital, Bucheon 420-767, Republic of Korea
}

Correspondence should be addressed to Geunbae Lim; limmems@postech.ac.kr and Hoon Lim; 43210@schmc.ac.kr

Received 3 December 2012; Revised 29 May 2013; Accepted 8 June 2013

Academic Editor: Jian Wei

Copyright (C) 2013 Seong J. Cho et al. This is an open access article distributed under the Creative Commons Attribution License, which permits unrestricted use, distribution, and reproduction in any medium, provided the original work is properly cited.

Fabrication of one-dimensional nanostructures is a key issue for optical devices, fluidic devices, and solar cells because of their unique functionalities such as antireflection and superhydrophobicity. Here, we report a novel one-step process to fabricate patternable hierarchical structures consisting of microstructures and one-dimensional nanostructures using a sacrificial layer. The layer plays a role as not only a micromask for producing microstructures but also as a nanomask for nanostructures according to the etching time. Using this method, we fabricated patterned hierarchical structures, with the ability to control the shape and density of the nanostructure. The various architectures provided unique functionalities. For example, our sacrificial-layer etching method allowed nanostructures denser than what would be attainable with conventional processes to form. The dense nanostructure resulted in a very low reflectance of the silicon surface (less than 1\%). The nanostructured surface and hierarchically structured surface also exhibited excellent antiwetting properties, with a high contact angle $\left(>165^{\circ}\right)$ and low sliding angle $\left(<1^{\circ}\right)$. We believe that our fabrication approach will provide new insight into functional surfaces, such as those used for antiwetting and antireflection surface applications.

\section{Introduction}

One-dimensional nanostructures are of great interest because of their unique properties. Examples found in nature have high surface-to-volume ratios and can impart antireflective and superhydrophobic behaviors [1-4]. Many attempts have been made to incorporate such functional nanostructures into various devices such as optical devices, solar cells, sensors, and biomedical devices to improve their performance or to impart special attributes [5-12]. The special functionalities of the nanostructured surfaces are governed by their architectures. For example, a high-aspect ratio nanostructured silicon surface can generate a graded refractive index and thereby minimize reflectivity over a wide range of wavelengths [13]. Also, by controlling the scale of a nanostructure, its surface reflectivity can be tuned while maintaining its superhydrophobic properties [5]. Making multiscale architectures is another key issue in the fabrication of functional nanostructures because their special geometries are crucial to their performance [14-17]. For example, the microstructures of a lotus leaf minimize contact area, and their nanostructures allow the surface to withstand very high pressures [14, 16]. However, in general, inefficient approaches, that is, those requiring two or more processes, are usually required to fabricate multiscale architectures.

We report here a simple, one-step fabrication method for multiscale silicon structures and the control of nanostructure morphology using a sacrificial layer. The patterned sacrificial layer protects the etching of silicon, resulting in the production of a micropattern early in the etching 


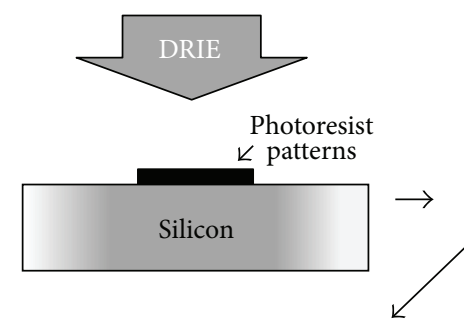

(a)

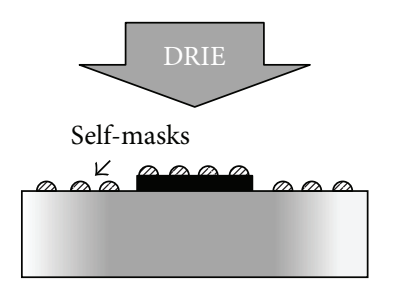

(b)

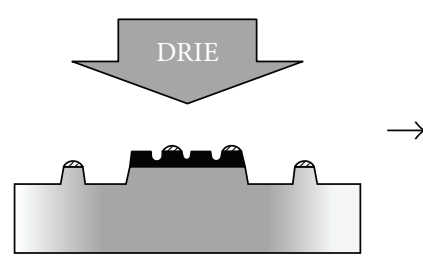

(c)

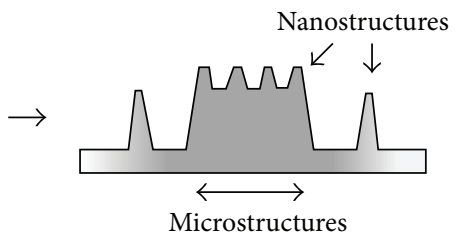

(d)

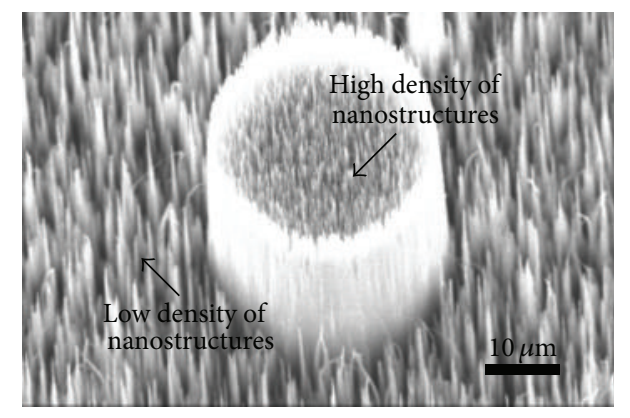

(e)

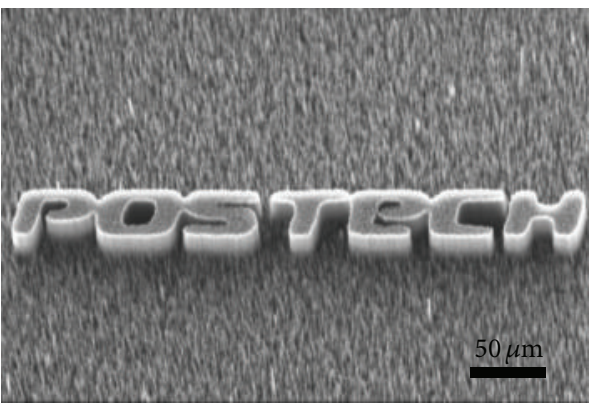

(f)

FIGURE 1: ((a)-(d)) Fabrication of patternable hierarchically structured silicon surfaces, using deep reactive-ion etching (DRIE) based on the "black silicon" method and using a sacrificial layer. ((e), (f)) Scanning electron microscopy (SEM) images of the hierarchical structures.

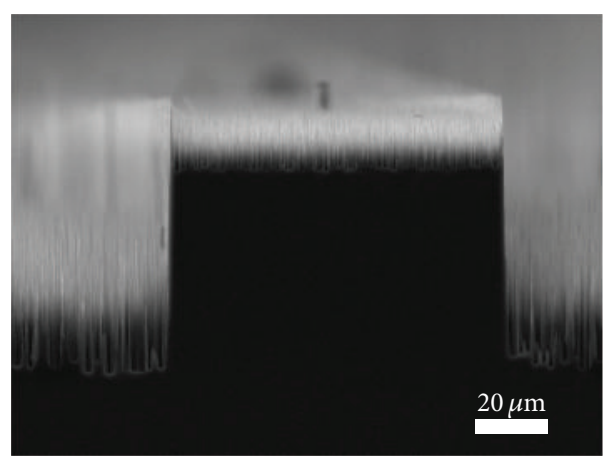

FIGURE 2: Cross-sectional SEM image of the hierarchical silicon structure.

process, whereas residue from the sacrificial layer, remaining after the final etching process, acts as a nanomask which produces the nanostructure. To verify the nanostructure formation, we analyzed the etching process morphologically and chemically using high-resolution scanning electron microscopy (HR-SEM) and energy dispersive spectrometry (EDS), respectively. Based on the method, we fabricated patternable hierarchical structures and controlled the shape and density of the nanostructure. In particular, a high-density nanostructure, six times denser than that produced using conventional procedures, had excellent antireflection properties (specular reflectance: average ca. $0.1 \%$; hemispherical reflectance: average ca. $1 \%$ ) over a broad range of wavelengths, compared with a polished silicon surface (specular reflectance: $\sim 40 \%$; hemispherical reflectance: $\sim 50 \%)$. The nanostructured surface and micropatterned surface also 


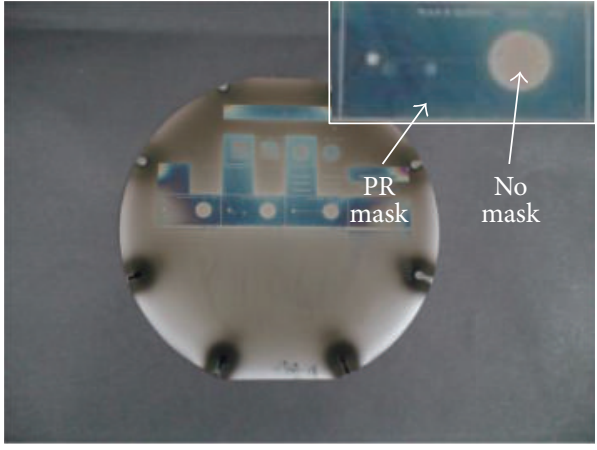

(a)

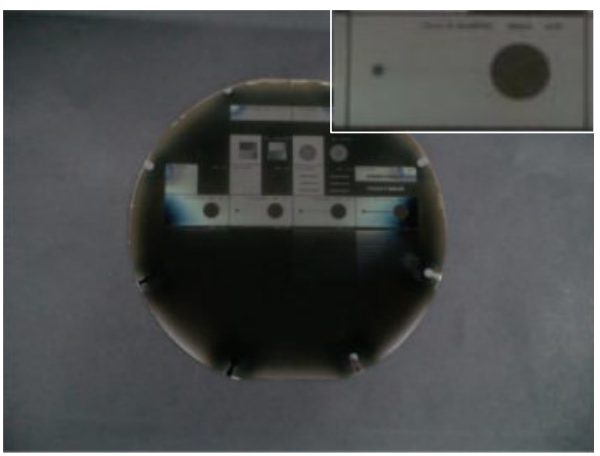

(c)

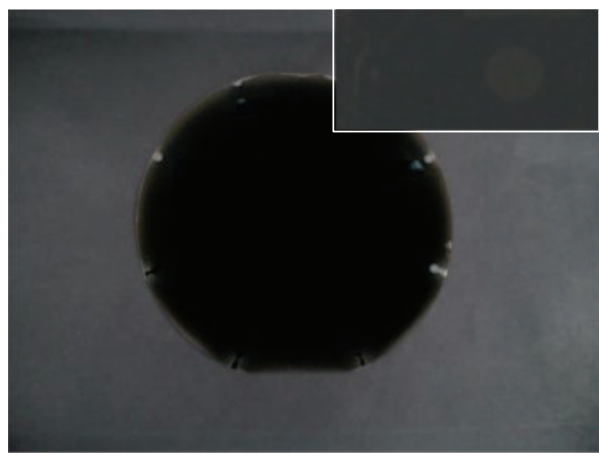

(e)
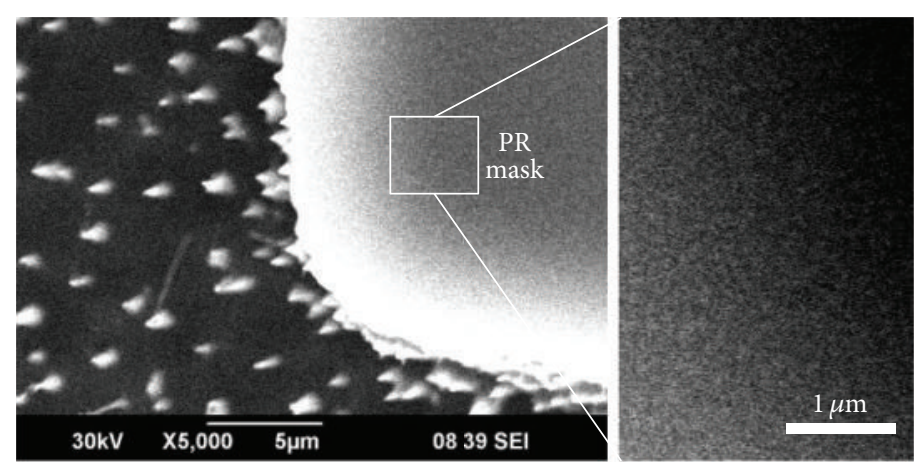

(b)
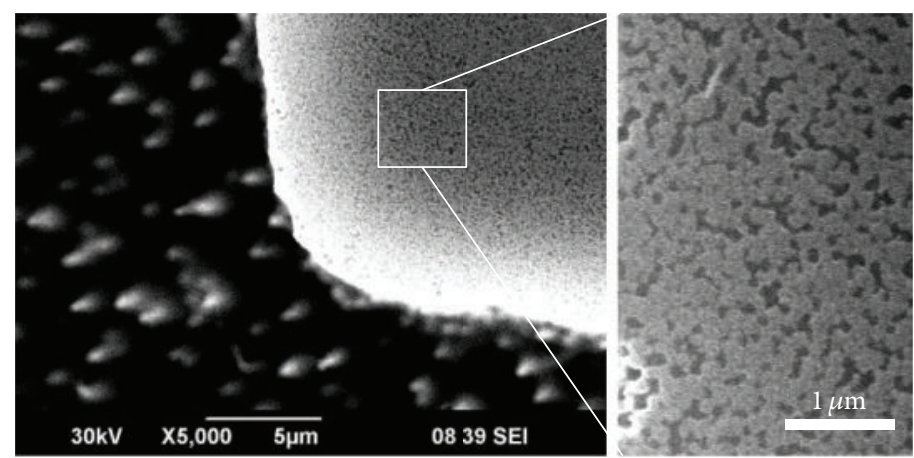

(d)
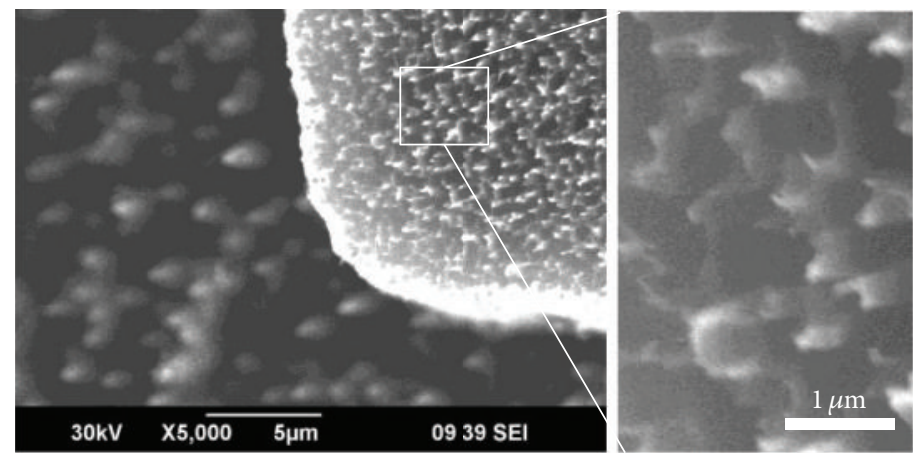

(f)

FIGURE 3: Optical images of nanostructured surfaces at various DRIE processing times: (a) $3000 \mathrm{~s}$, (c) $4000 \mathrm{~s}$, and (e) $5000 \mathrm{~s}$. (b), (d), and (f) are SEM images and magnified images of (a), (c), and (e), respectively.

exhibited stable antiwetting properties, with a high contact angle $\left(>165^{\circ}\right)$ and low sliding angle $\left(<1^{\circ}\right)$, which provided special functionality, such as self-cleaning.

\section{Experimental Section}

Figures 1(a)-1(d) illustrate the etching process using a sacrificial layer and shows SEM images of each step. First, cleaned and polished 4 in silicon wafers were prepared (P-type $<100>$ ), and photoresist (PR) (AZ 1512) patterns were fabricated on a silicon wafer as a sacrificial layer using the common photolithography process. Then, the wafer was etched by a deep reactive-ion etching (DRIE) process based on the "black silicon" method [5, 18-20]. During the black silicon process, micro-self-masks formed automatically on the silicon surface. The silicon surface was then anisotropically etched to form needle-shaped nanostructures called nanograss [5]. Detailed procedures are as follows: the prepared wafers were subjected to a pulsed etching process by an inductively coupled plasma multiplex system that used alternate cycles of etching and passivation (Surface Technology Systems, Ltd., $\mathrm{UK})$. In the etching cycle, sulfur hexafluoride $\left(\mathrm{SF}_{6}\right)$ and oxygen $\left(\mathrm{O}_{2}\right)$ gases flowed at $130 \mathrm{sccm}$ and $13 \mathrm{sccm}$, respectively. In the passivation cycle, the octafluorocyclobutane $\left(\mathrm{C}_{4} \mathrm{~F}_{8}\right)$ gas flow rate was $85 \mathrm{sccm}$. Coil power and platen power were $600 \mathrm{~W}$ and $30 \mathrm{~W}$, respectively. Switching times for the etching and passivation cycles were $6 \mathrm{~s}$ and $4 \mathrm{~s}$, respectively. The bias voltage was $120 \mathrm{~V}$ and $0 \mathrm{~V}$ for etching and passivation, respectively. Under these conditions, sparse nanograss having high aspect ratios (width: 50-3000 $\mathrm{nm}$ and 


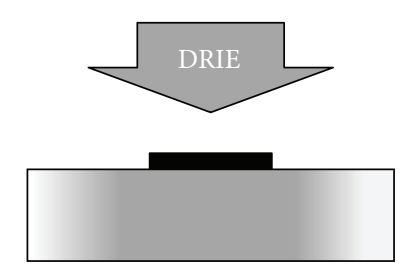

Flat photoresist pattern

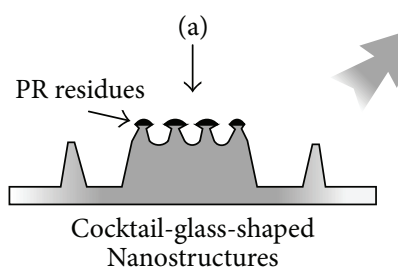

(b)

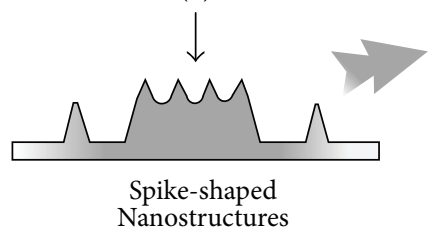

(c)

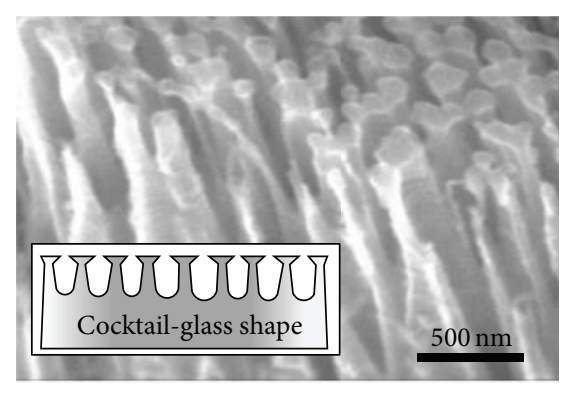

(d)

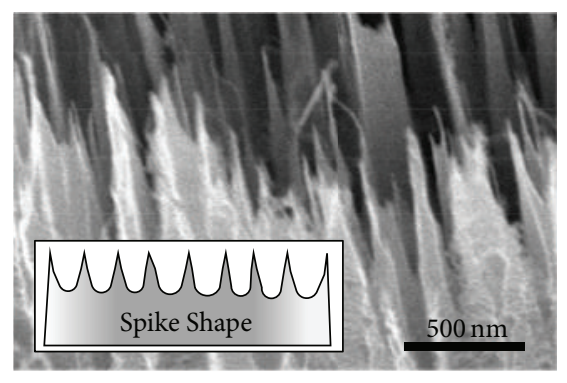

(e)

FIGURE 4: ((a)-(c)) The morphology of nanostructures was controlled by depositing a sacrificial layer and varying the process time.

(d) Cocktail-glass-shaped nanostructures. (e) Spike-shaped nanostructures.

length: $0.1-50 \mu \mathrm{m}$ ) formed. As the process time lengthened, the PR mask (thickness: $1.2 \mu \mathrm{m}$ ) was etched and additional nanograss formed.

SEM measurements were carried out with a fieldemission- (FE-) SEM instrument (SU-6600, Hitachi, Japan). Elemental composition of the nanostructure was analyzed using a Hitachi energy dispersive X-ray analysis (EDAX) energy spectra analyzer. Optical reflectance measurements of the nanostructured surface were conducted using an ultraviolet-visible-infrared (UV-Vis-IR) spectrophotometer (Cary 5000, Varian, USA), equipped with absolute specular and hemispherical reflectance accessories. Reflectance was measured from the UV range (wavelength: $200 \mathrm{~nm}$ ) to the near-IR range $(1000 \mathrm{~nm})$ to examine the effect of silicon nanostructures on broadband reflectance. Contact angles (CAs) were measured between $5 \mu \mathrm{L}$ deionized water droplets and the nanostructured surface, using a drop shape analysis system (DSA 100, Kruss, Germany) with the sessile drop method.

\section{Results and Discussion}

Patternable hierarchical silicon nanograss was fabricated using the black silicon process with PR patterns as a sacrificial layer, as shown in Figures 1(e) and 1(f). The patterned PR protected the silicon surface during the early stage DRIE process because of its high selectivity over silicon $(\sim 50)$ so that the microstructures produced would have the patterned shape. After long etching times (ca. $4000 \mathrm{~s}$ for a thickness of $1.2 \mu \mathrm{m}$ PR), the bare silicon was slightly etched to expose the bare silicon surface under the PR layer. At that time, nanograss began to form on the microstructure. The nanograss on the microstructure was denser than that on the unmasked surfaces. This phenomenon will be discussed later. Finally, patternable multiscale silicon structures were fabricated by the one-step etching process (etching time: $5000 \mathrm{~s}$ ). The height of the microstructure was about $57 \mu \mathrm{m}$, and the height of the nanostructure on the microstructure was about $15 \mu \mathrm{m}$ (Figure 2). The height of each structure could be controlled by the PR thickness and the etching time. A complex-shaped hierarchical structure was also fabricated (Figure 1(f)).

The shape of the nanograss on the microstructure changed as the etching process time increased, unlike that of the general nanograss structure. Figure 3 shows the shape changes that occurred on the silicon surface during the process. Just after the PR layer was removed (process time: ca. $4000 \mathrm{~s}$ ), partial etching of the silicon began, as evidenced by the formation of fine holes having a diameter of ca. $100 \mathrm{~nm}$ (Figure 3(d)). According to the cross-sectional view, as shown in Figures 4(d) and 4(e), the surface was etched in the form of a cocktail glass. This unique shape and the hierarchical structure are similar to those of gecko foot hair, which has a dry adhesive property (Figure 3(e)) [21, 22]. As the etching time increased, the tips of the silicon nanograss changed from the cocktail-glass shape to very sharp spikes (tip width: $<10 \mathrm{~nm}$ ), and the color of the surface turned from silver to black (Figures 3(a), 3(c), and 3(e)).

We assumed that the formation of this unique shape was due to residual PR (Figure 3(d)). This shape formed because intact PR prevented etching of the underlying silicon layer. Because of PR's nonreactive property to etching gas in the 


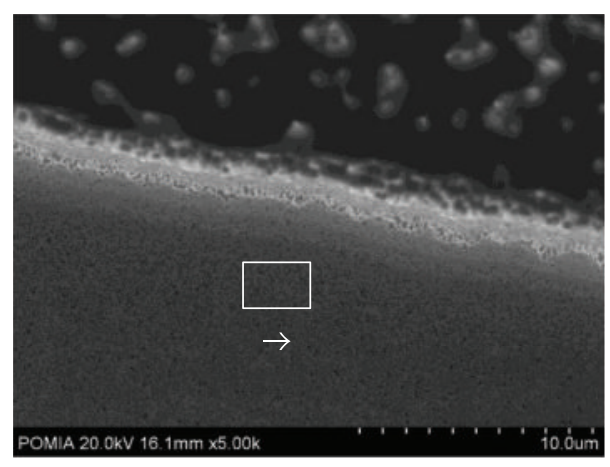

(a)

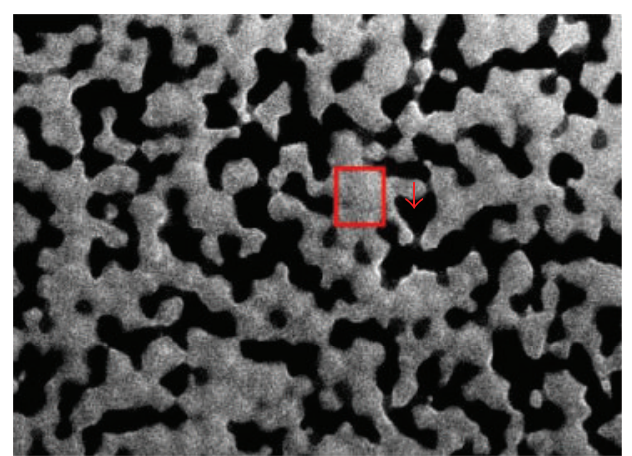

(b)

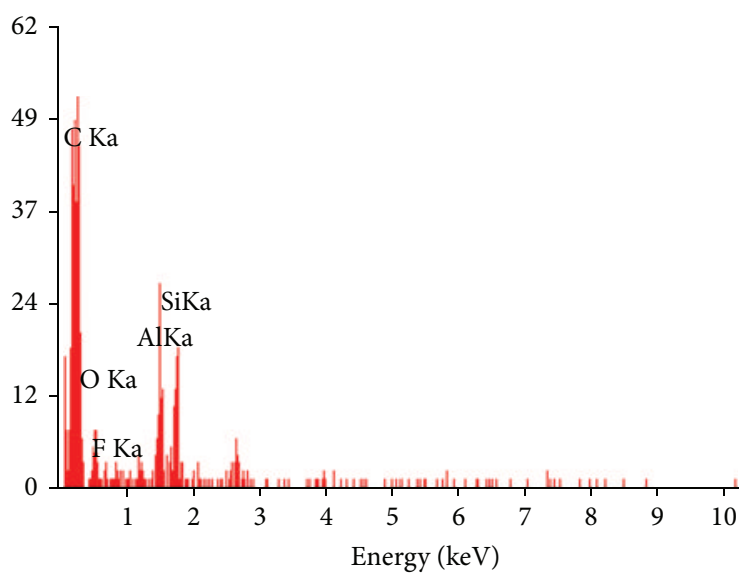

(c)

FIGURE 5: Energy dispersive spectrometry (EDS) analysis of the nanostructured surface fabricated by a sacrificial layer. (a) SEM image of the patterned hierarchical structure. (b) Magnified image of the high-density nanostructure on a micropatterned architecture in the early etching state. (c) EDS spectrum of the PR residue on the cocktail-glass-shaped nanostructures.

DRIE process, the PR residues could protect the silicon for a relatively long time to form such a cocktail-glass-shaped structure. To confirm the residual PR on silicon, we analyzed the composition of the cocktail-glass-shaped structure using EDS (Figure 5). As a result, carbon, which is the main element of PR but not present in silicon, existed on the surface of the etched structure.

The residual PR affected not only the shape of the silicon structure but also its density. The density difference can also be explained using a similar formation mechanism as that of the cocktail-glass-shaped structure. Conventional nanograss was formed by nonuniform etching of a thin passivation layer during the plasma etching process. Regarding the nanograss produced using our sacrificial-layer approach, the nonetched residual $\mathrm{PR}$ enhanced the passivation process, because it was more solid and thicker than the fluorocarbon polymer that was used as a passivation layer for the conventional plasma etching process. Thus, denser nanomasks could be formed on silicon to increase the density of the nanograss. Consequently, the nanograss density could be modified using a sacrificial layer. Hierarchical structures with various densities were fabricated using this process. Figure 6 shows two kinds of hierarchical structures, one with a high density for the nanostructure (Figure 6(a), nanostructure density:
$19.8 \times 10^{7} \mathrm{~cm}^{-2}$ ) and the other with a low density for the nanostructure (Figure $6(\mathrm{~b}), 2.9 \times 10^{7} \mathrm{~cm}^{-2}$ ). The high-density nanostructures were fabricated using our sacrificial-layer method. The low-density structure could be easily fabricated: microstructures were made using a PR pattern, and then the $\mathrm{PR}$ layer was removed with $\mathrm{O}_{2}$ plasma (10 min); the nanograss formed on the cleaned microstructure. This suggests that residual PR was responsible for the nanograss density.

The marked difference in densities influenced the optical properties of the nanostructure. In general, the reflectance tendency of a subwavelength structure is determined by its refractive index profile [5]. The effective index of the subwavelength composite structure depends on its volume fraction and its intrinsic refractive index. Thus, the density of nanograss, which is related to both the volume fraction and the refractive index, played a crucial role in reflectance. Specifically, in the case of sparse nanograss (low density), a gradual increase in the refractive index profile was generated at the top and middle regions, due to its sharp, fine architecture; however, at the bottom, the index had changed dramatically due to the sparse architecture. The dense nanograss, on the other hand, generated a graded profile over the entire region due to the dense architecture. Figures 6(c) and 6(d) show how the specular and hemispherical reflectance 


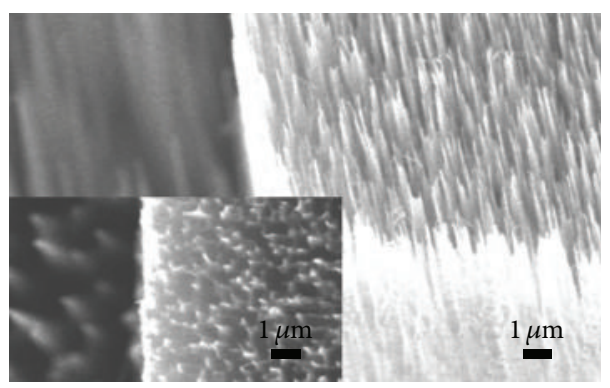

(a)

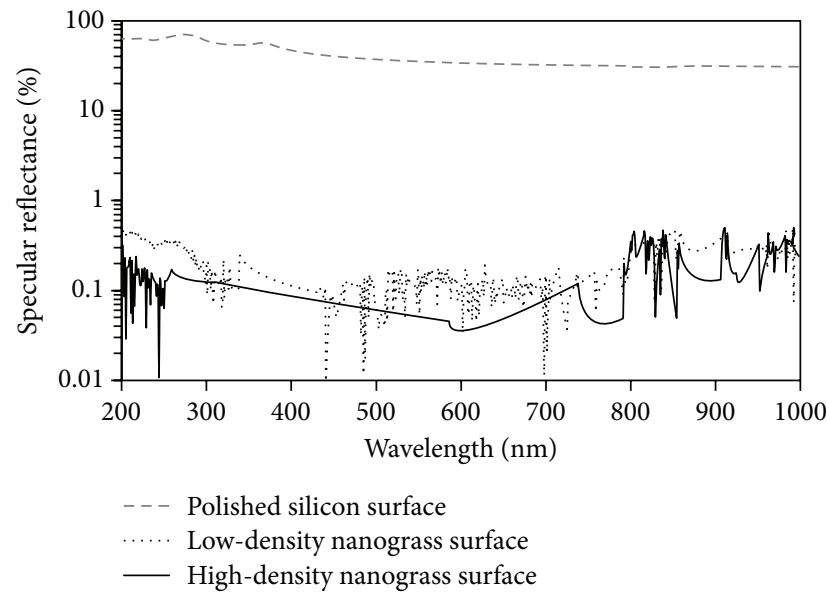

(c)

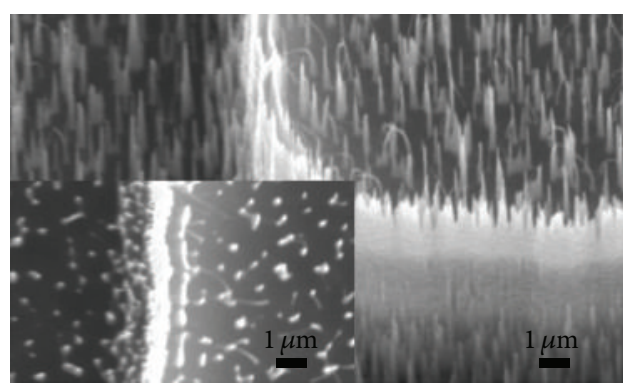

(b)

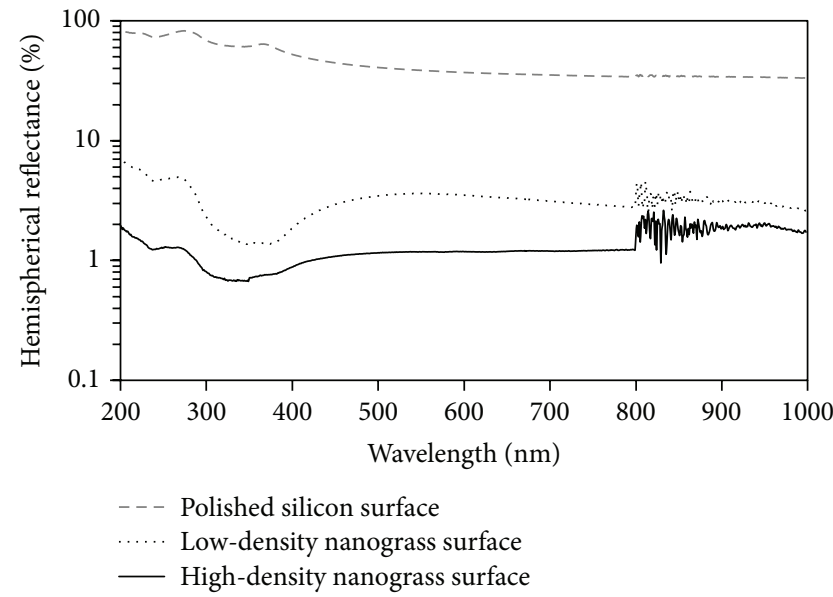

(d)

FIGURE 6: Control of the nanostructure density and measurement of optical properties. ((a), (b)) Hierarchical structures having high density and low density, respectively. ((c), (d)) Surface with antireflectivity over a broad wavelength region; the specular reflection and diffuse reflection are suppressed by the density of the nanostructure.

varied with the density of the nanostructure, respectively. The nanostructured silicon surface exhibited outstanding antireflectance compared with the polished silicon surface. In particular, the high-density nanograss surface provided excellent antireflective surfaces that possessed ca. $0.1 \%$ specular reflectance and ca. $1 \%$ hemispherical reflectance over the entire ultraviolet-visible-near infrared range (200-1000 nm). This reflectance is less than that for low-density nanograss surfaces (average specular reflectance of ca. $0.3 \%$ and average hemispherical reflectance of ca. 3\%) and much lower than that for polished silicon (average of ca. 40\%).

Fabrication of a hierarchical structure has been one of the main issues in antiwettable surface research. The antiwettability, which is often referred to as superhydrophobicity, can be achieved by low-energy chemical compositions and geometric micro- and nanostructured surfaces. To produce an antiwetting surface, we fabricated the nanostructured surface and hierarchical structure using our sacrificial-layer process. The surfaces were deposited with a low surface energy fluorocarbon polymer layer by a plasma-polymerized fluorocarbon coating (PPFC), using the same system as the DRIE process. Figure 7 shows the wettability of a highdensity nanograss surface and a micro/nanohierarchically structured surface with a $5 \mu \mathrm{L}$ sessile water drop. As a result, the surface exhibited excellent superhydrophobicity with a high water contact angle (CA) and low sliding angle (SA). The high-density nanograss had a static CA of $165^{\circ}$ and $\mathrm{SA}$ of $<1^{\circ}$. The micro/nanohierarchically structured surface showed more outstanding antiwetting properties $\left(\mathrm{CA} \sim 170^{\circ}\right.$ and $\mathrm{SA}<1^{\circ}$ ). The air gap between the drop and the surface (white arrow in the Figure 7) was observed optically. The air gap provides strong evidence for the formation of a stable, superhydrophobic surface with a Cassie state, in which a liquid droplet rests on a composite surface composed of a solid and trapped air.

\section{Conclusion}

We have demonstrated a novel process for the fabrication of patternable hierarchical structures that are a combination of a microstructure and a one-dimensional nanostructure. The morphologies of these structures have been modified using a PR sacrificial layer. A micropatterned hierarchical structure having a complex shape could be fabricated by a one-step process without invoking a nanopatterning process. The nanostructure can be controlled to have a cocktailglass shape that resembled a gecko's foot hair or sharp spike shape having a high-aspect ratio. Our sacrificial-layer etching method allows denser nanostructures to form than would be attainable with existing processes. The dense nanostructure 


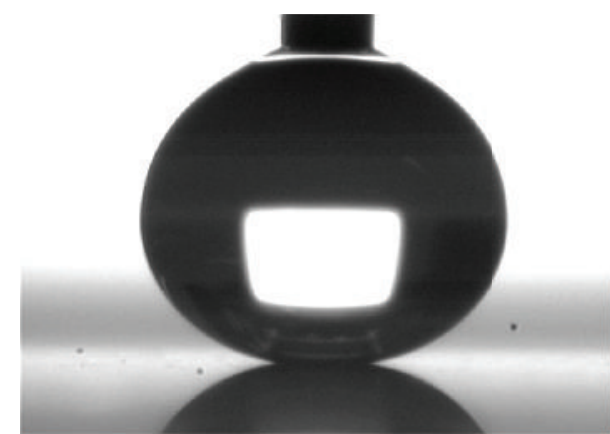

(a)

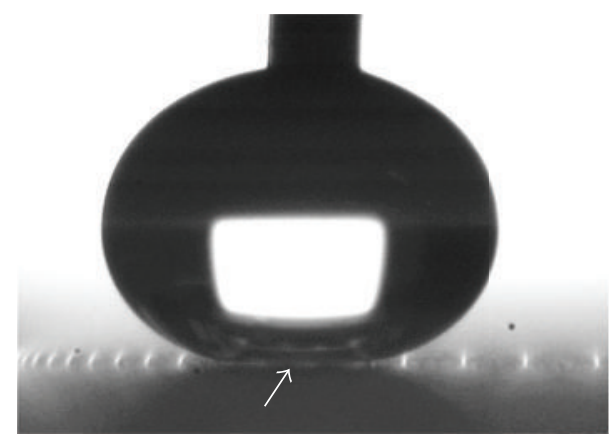

(b)

Figure 7: (a) Antiwettability test of the high-density nanograss surface with a $5 \mu \mathrm{L}$ sessile water drop (static water contact angle $=165^{\circ}$ and sliding angle $<1^{\circ}$ ). (b) Antiwettability test of a micro/nanohierarchically structured surface (static water contact angle $=170^{\circ}$ and sliding angle $<1^{\circ}$ ). The air gap between the drop and the surface (white arrow) was observed optically, due to the hierarchical surface's superior antiwettability.

resulted in a very low reflectance of the silicon surface, less than $1 \%$. The nanostructured surface and hierarchically structured surface also exhibited excellent antiwetting properties. We believe our fabrication method can be utilized to prepare various functional surfaces, such as antireflecting surfaces for high-performance optical devices (e.g., solar cells) and selfcleaning, superhydrophobic surfaces.

\section{Conflict of Interests}

The authors declare no financial conflict of interests.

\section{Acknowledgments}

This research was financially supported by a grant to MEMS Research Center for National Defense funded by Defense Acquisition Program Administration.

\section{References}

[1] Y. Li, J. Zhang, and B. Yang, "Antireflective surfaces based on biomimetic nanopillared arrays," Nano Today, vol. 5, no. 2, pp. $117-127,2010$

[2] Y.-F. Huang, S. Chattopadhyay, Y.-J. Jen et al., "Improved broadband and quasi-omnidirectional anti-reflection properties with biomimetic silicon nanostructures," Nature Nanotechnology, vol. 2, no. 12, pp. 770-774, 2007.

[3] C. Dorrer and J. Rühe, "Wetting of silicon nanograss: from superhydrophilic to superhydrophobic surfaces," Advanced Materials, vol. 20, no. 1, pp. 159-163, 2008.

[4] K. T. Lee and J. Cho, "Roles of nanosize in lithium reactive nanomaterials for lithium ion batteries," Nano Today, vol. 6, no. 1, pp. 28-41, 2011.

[5] S. J. Cho, T. An, J. Y. Kim, J. Sung, and G. Lim, "Superhydrophobic nanostructured silicon surfaces with controllable broadband reflectance," Chemical Communications, vol. 47, no. 21, pp. 6108-6110, 2011.

[6] K. Q. Peng and S. T. Lee, "Silicon nanowires for photovoltaic solar energy conversion," Advanced Materials, vol. 23, no. 2, pp. 198-215, 2011.
[7] Z. Fan, H. Razavi, J.-W. Do et al., “Three-dimensional nanopillar-array photovoltaics on low-cost and flexible substrates," Nature Materials, vol. 8, no. 8, pp. 648-653, 2009.

[8] T. An, W. Choi, E. Lee, I. T. Kim, W. Moon, and G. Lim, "Fabrication of functional micro- and nanoneedle electrodes using a carbon nanotube template and electrodeposition," Nanoscale Research Letters, vol. 6, article 306, 2011.

[9] W. Choi, T. An, and G. Lim, "Organic electrochemical transistors based on a dielectrophoretically aligned nanowire array," Nanoscale Research Letters, vol. 6, article 339, 2011.

[10] T. An, W. Choi, S. W. Joo, and G. Lim, "A novel fabrication method of CNT-CP composite single nanowires self-templated by dielectrophoresis and electropolymerization," Physica Status Solidi (RRL), vol. 5, no. 7, pp. 235-237, 2011.

[11] S. J. Cho, B. Kim, T. An, and G. Lim, "Replicable multilayered nanofibrous patterns on a flexible film," Langmuir, vol. 26, no. 18, pp. 14395-14399, 2010.

[12] S. J. Cho, H. Nam, T. An, and G. Lim, "Replicable and shapecontrollable fabrication of electrospun fibrous scaffolds for tissue engineering," Journal of Nanoscience and Nanotechnology, vol. 12, no. 12, pp. 9047-9050, 2012.

[13] S. Chattopadhyay, Y. F. Huang, Y. J. Jen, A. Ganguly, K. H. Chen, and L. C. Chen, "Anti-reflecting and photonic nanostructures," Materials Science and Engineering: R, vol. 69, pp. 1-35, 2010.

[14] Y. Su, B. Ji, K. Zhang, H. Gao, Y. Huang, and K. Hwang, "Nano to micro structural hierarchy is crucial for stable superhydrophobic and water-repellent surfaces," Langmuir, vol. 26, no. 7, pp. 4984-4989, 2010.

[15] Y. Xiu, L. Zhu, D. W. Hess, and C. P. Wong, "Hierarchical silicon etched structures for controlled hydrophobicity/ superhydrophobicity," Nano Letters, vol. 7, no. 11, pp. 3388-3393, 2007.

[16] T. An, S. J. Cho, W. Choi, J. H. Kim, S. T. Lim, and G. Lim, "Preparation of stable superhydrophobic mesh with a biomimetic hierarchical structure," Soft Matter, vol. 7, no. 21, pp. 9867-9870, 2011.

[17] S. J. Cho, H. Nam, H. Ryu, and G. Lim, "A rubberlike stretchable fibrous membrane with anti-wettability and gas breathability," Advanced Functional Materials, 2013.

[18] H. Jansen, M. de Boer, R. Legtenberg, and M. Elwenspoek, "The black silicon method: a universal method for determining the parameter setting of a fluorine-based reactive ion etcher in deep silicon trench etching with profile control," Journal of 
Micromechanics and Microengineering, vol. 5, no. 2, pp. 115-120, 1995.

[19] M. Gharghi and S. Sivoththaman, "Formation of nanoscale columnar structures in silicon by a maskless reactive ion etching process," Journal of Vacuum Science and Technology A, vol. 24, no. 3, pp. 723-727, 2006.

[20] I. I. Amirov and N. V. Alov, "Formation of microstructures on silicon surface in a fluorinated plasma via the cyclic etchingpassivation process," High Energy Chemistry, vol. 42, no. 2, pp. 132-136, 2008.

[21] H. E. Jeong, J. K. Lee, H. N. Kim, S. H. Moon, and K. Y. Suh, "A nontransferring dry adhesive with hierarchical polymer nanohairs," Proceedings of the National Academy of Sciences of the United States of America, vol. 106, no. 14, pp. 5639-5644, 2009.

[22] Z. L. Wang, "Nanomaterials: sticky but not messy," Nature Nanotechnology, vol. 4, pp. 407-408, 2009. 

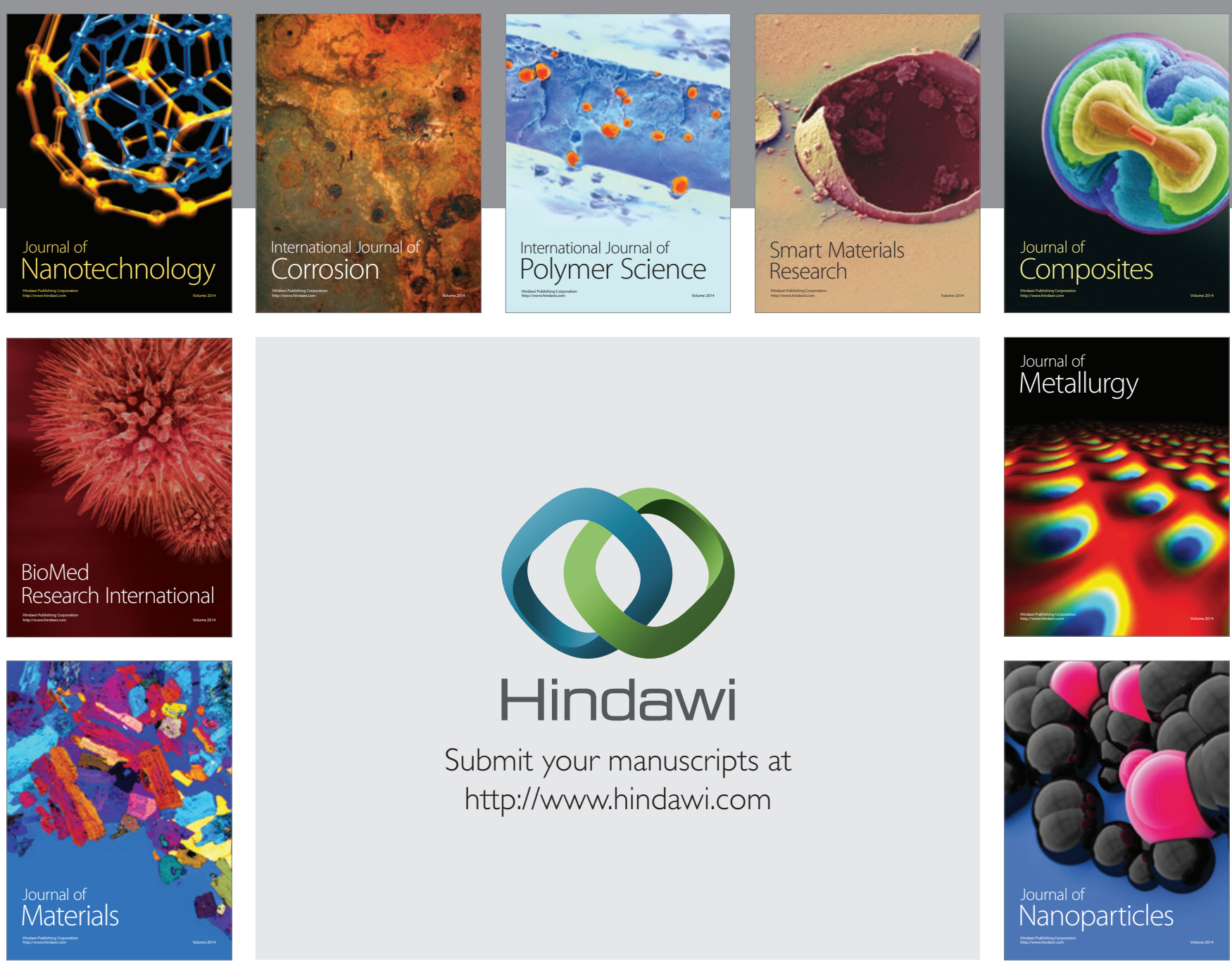

Submit your manuscripts at http://www.hindawi.com
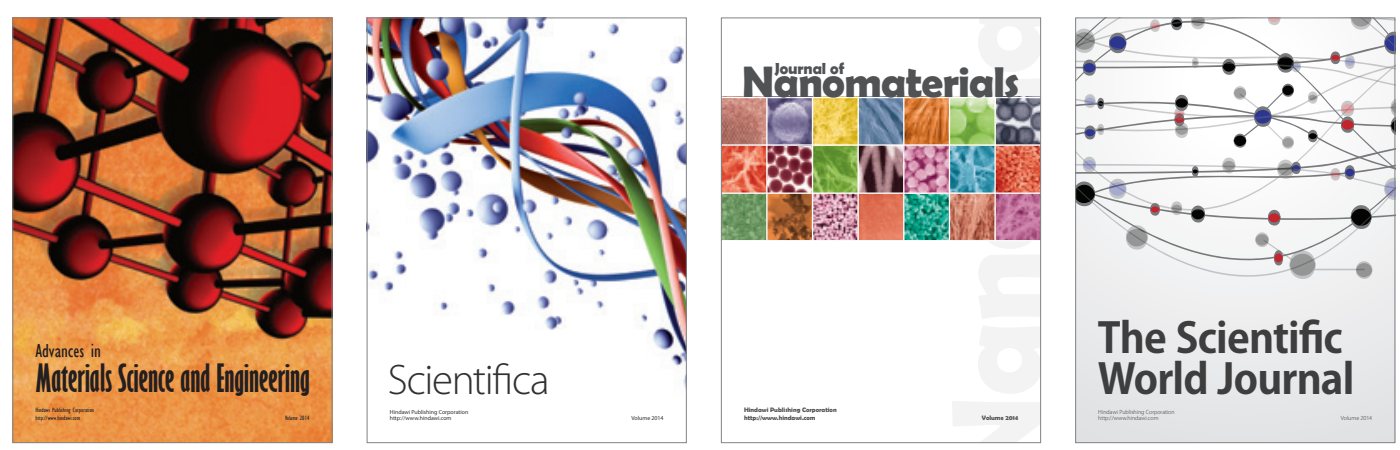

\section{The Scientific World Journal}
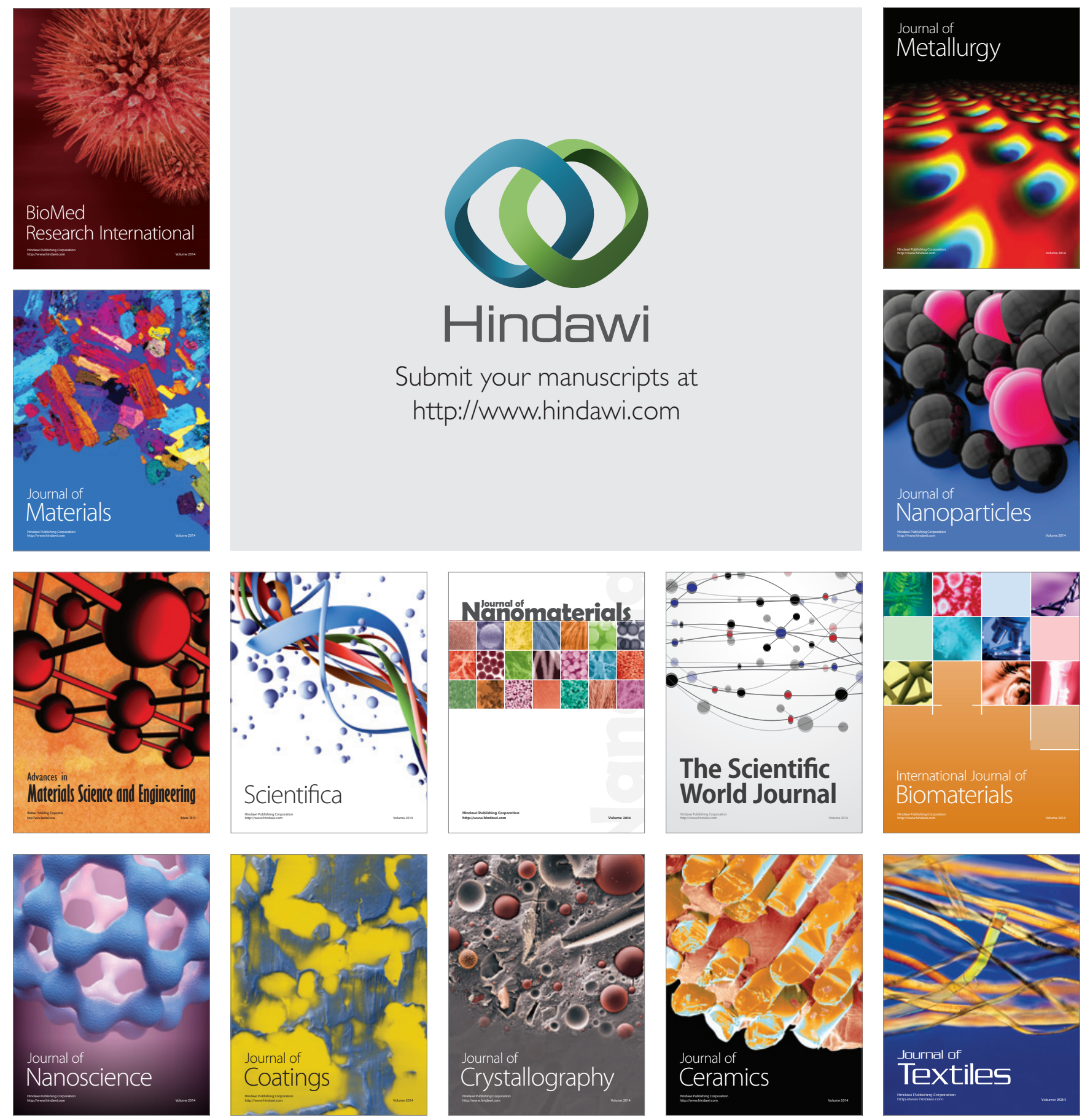